\title{
ANALYSIS AND CORRECTION OF SYSTEMATIC HEIGHT MODEL ERRORS
}

\author{
K. Jacobsen \\ Leibniz University Hannover, Institute of Photogrammetry and Geoinformation, Germany; \\ jacobsen@ipi.uni-hannover.de
}

Commission I, WG I/4

KEY WORDS: DSM/DTM, systematic error, optical space images, orientation, satellite jitter

\begin{abstract}
:
The geometry of digital height models (DHM) determined with optical satellite stereo combinations depends upon the image orientation, influenced by the satellite camera, the system calibration and attitude registration. As standard these days the image orientation is available in form of rational polynomial coefficients (RPC). Usually a bias correction of the RPC based on ground control points is required. In most cases the bias correction requires affine transformation, sometimes only shifts, in image or object space. For some satellites and some cases, as caused by small base length, such an image orientation does not lead to the possible accuracy of height models. As reported e.g. by Yong-hua et al. 2015 and Zhang et al. 2015, especially the Chinese stereo satellite ZiYuan-3 (ZY-3) has a limited calibration accuracy and just an attitude recording of $4 \mathrm{~Hz}$ which may not be satisfying. Zhang et al. 2015 tried to improve the attitude based on the color sensor bands of ZY-3, but the color images are not always available as also detailed satellite orientation information. There is a tendency of systematic deformation at a Pléiades tri-stereo combination with small base length. The small base length enlarges small systematic errors to object space. But also in some other satellite stereo combinations systematic height model errors have been detected. The largest influence is the not satisfying leveling of height models, but also low frequency height deformations can be seen.

A tilt of the DHM by theory can be eliminated by ground control points (GCP), but often the GCP accuracy and distribution is not optimal, not allowing a correct leveling of the height model. In addition a model deformation at GCP locations may lead to not optimal DHM leveling. Supported by reference height models better accuracy has been reached. As reference height model the Shuttle Radar Topography Mission (SRTM) digital surface model (DSM) or the new AW3D30 DSM, based on ALOS PRISM images, are satisfying. They allow the leveling and correction of low frequency height errors and lead to satisfying correction of the DSM based on optical satellite images.

The potential of DHM generation, influence of systematic model deformation and possibilities of improvement has been investigated.
\end{abstract}

\section{INTRODUCTION}

Systematic height model errors by model tilt and low frequency systematic errors often can be seen in height models based on very high resolution optical satellites. In most cases a not satisfying number and distribution of GCP is available to correct systematic height model errors. Free of charge available reference height models allow a correction of such errors. SRTM DSM is often used for correction. With the new AW3D30 DSM an improvement against SRTM DSM is available. Also in the Near East where SRTM is available only with 3 arcsec point spacing ( $\sim 90 \mathrm{~m}$ at the equator), AWD3D30 has 1arcsec point spacing and usually a higher accuracy as SRTM. ALOS PRISM was active from January 2006 up to May 2011, corresponding to this the AW3D30 DSM is more actual as the SRTM DSM based on information from year 2000.

Horizontal shifts of height models in areas with not well known geodetic datum have to be determined and respected by comparison of height models. In the following investigation this was required for the test area Zonguldak. An automatic orientation as d'Angelo (2013) describes for large blocks satellite image blocks was not possible in the flat test area Warsaw, in addition low frequency height errors have to be determined and respected for the individual height models. Even if the systematic errors sometimes have only limited influence to the accuracy numbers, it should be respected to avoid local discrepancies.

\section{USED DATA SETS}

In cooperation with the Chinese Satellite Surveying and Mapping Application Centre (SASMAC) an ISPRS benchmark test for ZY-3 has been set up in Sainte-Maxime, France, (http://www.isprs.org/data/zy-3/data/referencedata/Default.asps ). Ground control points and a reference DSM based on aerial photogrammetric survey have been supported by the French National Institute of Geographic and Forest Information (IGN). The GCP as well as the DSM have been transformed to UTM coordinate system and the DSM has been reduced to $10 \mathrm{~m}$ point spacing.

The optical tri-stereo satellite ZY-3 has a backward and a forward view with $3.4 \mathrm{~m}$ ground sampling distance (GSD) and a nadir view with $2.1 \mathrm{~m}$ GSD. The base to height relation for the backward and forward combination is 1:1.15. Only the results based on backward and forward scenes for ZY3 and Pléiades tri-stereo combinations are discussed below.

The test area of the Bülent Ecevit University in Zonguldak, Turkey, is covered by a tri-stereo arrangement of Pléiades images. The used image triplet has a very short base length corresponding to an angle of convergence between the first and last image of $12.6^{\circ}$. The view direction with $4.7^{\circ}$ across orbit is close to nadir view, so the geometric ground sampling distance is ranging from $72.6 \mathrm{~cm} \times 72.0 \mathrm{~cm}$ to $69.9 \mathrm{~cm} \times 71.1 \mathrm{~cm}$ (Jacobsen and Topan 2015). The short base length has the advantage of very similar images, leading to good matching results. The disadvantage of height determination with short base length is 
nearly compensated by the better automatic image matching of the images being more similar as in case of a large base. But systematic image errors are enlarged by the small base to height relation of 1:4.5.

In addition a Cartosat- 1 stereo scene of the Warsaw test area has been investigated. Cartosat-1 has 2.5m GSD and a base to height relation of 1:1.6.

All generated height models have been compared with reference height models of satisfying accuracy. Of course under operational conditions such reference information is not available, requiring free of charge available height models even with lower accuracy, which can be used for the correction of systematic height errors.

\section{IMAGE ORIENTATION}

For all used data sets the geo-reference is available by rational polynomial coefficients (RPC), presenting the relation between geographic object coordinates and the image positions by the ratio of third order polynomials (Grodecki 2001). The RPC have a limited absolute accuracy corresponding to the quality of the direct sensor orientation. An improvement by image orientation using GCP or a reference height model usually is required. Without pre-correction by GCP the absolute position of the height model, generated by image matching of the stereo combination, in most cases is not satisfying. As usual, the orientation of the used Hannover program RAPORIO (Jacobsen 2003) includes the possibility of bias correction based on GCP up to affinity transformation and even an additional correction of the view direction if enough GCP with larger height differences are available. The bias correction is supported by significance check of the individual affinity parameters. The orientation is done individually for any image. By intersection of corresponding image points, based on the determined orientation parameters, three-dimensional ground coordinates can be computed, allowing also a check of the height model leveling. Even if the same GCP are used, the bias correction by affinity transformation, being independent for both used images, may influence the height model leveling.

Differences of the scale parameters in flight direction may cause a height model tilt in flight direction, while differences of the angular affinity may cause a tilt across flight direction. Low frequency height errors cannot be caused by bias correction with affine transformation.

\begin{tabular}{|l|c|c|c|c|}
\hline 12 GCP & \multicolumn{2}{|c|}{ Bias correction affine } & \multicolumn{2}{c|}{ Bias correction shift } \\
\hline View $\backslash$ & SX & SY & SX & SY \\
\hline forward & $1.89 \mathrm{~m}$ & $1.81 \mathrm{~m}$ & $3.17 \mathrm{~m}$ & $2.67 \mathrm{~m}$ \\
\hline backward & $1.91 \mathrm{~m}$ & $2.51 \mathrm{~m}$ & $3.26 \mathrm{~m}$ & $3.07 \mathrm{~m}$ \\
\hline
\end{tabular}

Table 1. Standard deviations of ZY-3 GCP based on RPCimage orientations improved by bias correction with affine transformation respectively shift; test area Sainte-Maxime

\begin{tabular}{|c|c|c|c|c|c|}
\hline \multicolumn{3}{|c|}{ Bias correction affine } & \multicolumn{3}{c|}{ Bias correction shift } \\
\hline SX & SY & SZ & SX & SY & SZ \\
\hline $1.78 \mathrm{~m}$ & $1.67 \mathrm{~m}$ & $3.24 \mathrm{~m}$ & $2.85 \mathrm{~m}$ & $2.72 \mathrm{~m}$ & $3.87 \mathrm{~m}$ \\
\hline
\end{tabular}

Table 2. 3D-determination of ground control points $\mathrm{ZY}-3$

\begin{tabular}{|l|c|c|c|c|}
\hline 170 GCP & \multicolumn{2}{|c|}{ Bias correction affine } & \multicolumn{2}{c|}{ Bias correction shift } \\
\hline View $\backslash$ & SX & SY & SX & SY \\
\hline forward & $0.44 \mathrm{~m}$ & $0.48 \mathrm{~m}$ & $0.45 \mathrm{~m}$ & $0.50 \mathrm{~m}$ \\
\hline backward & $0.44 \mathrm{~m}$ & $0.48 \mathrm{~m}$ & $0.44 \mathrm{~m}$ & $0.50 \mathrm{~m}$ \\
\hline
\end{tabular}

Table 3. Standard deviations of Pléiades 1A GCP based on RPC-image orientations improved by bias correction with affine transformation respectively shift; test area Zonguldak

\begin{tabular}{|c|c|c|c|c|c|}
\hline \multicolumn{3}{|c|}{ Bias correction affine } & \multicolumn{3}{c|}{ Bias correction shift } \\
\hline SX & SY & SZ & SX & SY & SZ \\
\hline $0.43 \mathrm{~m}$ & $0.49 \mathrm{~m}$ & $1.32 \mathrm{~m}$ & $0.43 \mathrm{~m}$ & $0.52 \mathrm{~m}$ & $1.33 \mathrm{~m}$ \\
\hline
\end{tabular}

Table 4. 3D-determination of ground control points, Pléiades, first and last image; Zonguldak

\begin{tabular}{|l|c|c|c|c|}
\hline 33 GCP & \multicolumn{2}{|c|}{ Bias correction affine } & \multicolumn{2}{c|}{ Bias correction shift } \\
\hline View $\backslash$ & SX & SY & SX & SY \\
\hline forward & $1.35 \mathrm{~m}$ & $1.27 \mathrm{~m}$ & $12.54 \mathrm{~m}$ & $2.83 \mathrm{~m}$ \\
\hline backward & $1.41 \mathrm{~m}$ & $1.50 \mathrm{~m}$ & $16.86 \mathrm{~m}$ & $1.64 \mathrm{~m}$ \\
\hline
\end{tabular}

Table 5. Standard deviations of Cartosat-1 GCP based on RPCimage orientations improved by bias correction with affine transformation respectively shift; test area Warsaw

\begin{tabular}{|c|c|c|c|c|c|}
\hline \multicolumn{3}{|c|}{ Bias correction affine } & \multicolumn{3}{c|}{ Bias correction shift } \\
\hline SX & SY & SZ & SX & SY & SZ \\
\hline $1.33 \mathrm{~m}$ & $1.42 \mathrm{~m}$ & $1.86 \mathrm{~m}$ & $13.79 \mathrm{~m}$ & $2.69 \mathrm{~m}$ & $4.71 \mathrm{~m}$ \\
\hline
\end{tabular}

Table 6. 3D-determination of ground control points, Cartosat-1; test area Warsaw

\begin{tabular}{|c|c|c|c|}
\hline $\begin{array}{c}\text { Tilt X } \\
\text { (bias shift) }\end{array}$ & $\begin{array}{c}\text { Tilt Y } \\
\text { (bias shift) }\end{array}$ & $\begin{array}{c}\text { Tilt } X \\
\text { (bias affine) }\end{array}$ & $\begin{array}{c}\text { Tilt Y } \\
\text { (bias affine) }\end{array}$ \\
\hline \multicolumn{4}{|c|}{ ZY3 Sainte-Maxime range X: 55km range Y: 39km (12 GCP) } \\
\hline $3.57 \mathrm{~m}$ & $3.43 \mathrm{~m}$ & $0.55 \mathrm{~m}$ & 0.2 \\
\hline \multicolumn{4}{|c|}{ Pléiades Zonguldak range X: 20km range Y: 17km (170 GCP) } \\
\hline $1.16 \mathrm{~m}$ & $1.04 \mathrm{~m}$ & $0.70 \mathrm{~m}$ & $0.82 \mathrm{~m}$ \\
\hline \multicolumn{4}{|c|}{ ange $\mathrm{X}: 13 \mathrm{~km}$ range $\mathrm{Y}: 31 \mathrm{~km}$ (33 GCP) } \\
\hline $8.07 \mathrm{~m}$ & $1.46 \mathrm{~m}$ & $0.39 \mathrm{~m}$ & $-0.03 m$ \\
\hline
\end{tabular}

Table 7. Influence of model tilt over covered range determined at control points

The number of unknowns for bias correction has a limited influence to Pléiades orientation; while it is very important for Cartosat-1 and should not be neglected for ZY3 (tables 1 - 7). The bias correction is influencing especially the Cartosat- 1 data, while it is also clearly improving ZY-3 data but can be neglected for Pléiades data. The model tilt determined by $\mathrm{Z}$ coordinates of the GCP is clearly smaller if the RPCorientations are determined by bias correction with affine transformation as in case of only a shift correction. Of course the quality of the model tilt determination depends upon the number and distribution of the GCP. In all three cases the distribution is satisfying, but the number of GCP with 12, 170 respectively 33 is varying. The achieved orientation accuracy has to be seen in relation to the ground sampling distance and the height the base to height relation even if the vertical accuracy is not linear depending upon it (Jacobsen et al. 2014).

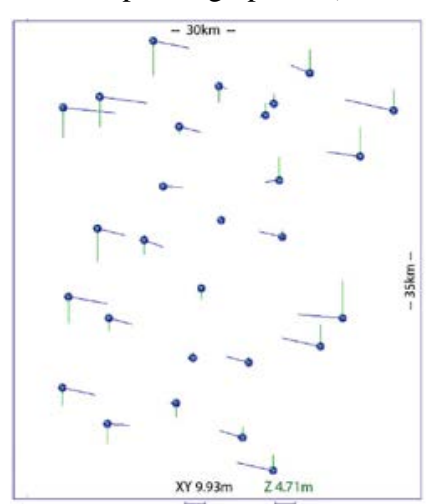

Figure 1. Discrepancies at check points, Cartosat-1, Warsaw, based on RPC-orientation with bias correction by shift, green vectors $($ vertical direction $)=$ height discrepancies 
The requirement of the bias correction by affine transformation is clearly demonstrated at figure 1 . The $\mathrm{X}$-direction shows an affine deformation and the Z-discrepancies are dominated by a model tilt.

\section{ANALYSIS OF HEIGHT MODELS}

Digital surface models have been generated by least squares matching with region growing. A possible DSM-shift in X-and Y-direction has been checked by adjustment with Hannover program DEMSHIFT. Due to not well known datum of the Turkish coordinate system for the data set Zonguldak it was necessary to shift the DSM in X-and Y-direction. So the following height model analysis is not influenced by horizontal shifts.

For ZY3 as reference a DSM from high resolution aerial images, made by IGN France, is available and for Pléiades a data set manually measured in large scale aerial images with points on the bare ground, but with free view to the ground. For Cartosat a reference DTM from Polish survey administration is given. The analysis of Warsaw test area is based only on open areas, in addition the data have been filtered for points not belonging to bare ground. So in general the influence of vegetation is limited. In Saintes-Maxime the tendency of a vegetation change can be seen. In addition the AW3D30 DSM (from ALOS PRISM) (Takaku et al., 2014) has been used for an elimination of the systematic height differences.

The systematic height errors of the ZY3 DSM, based on orientation by bias correction with affine transformation, against the reference DSM from IGN (figure 2) are obvious - in the upper part the orange tone in the lower part the blue tone is dominating, corresponding to a rotation of the DSM in Ydirection by $-9.67 \mathrm{~m}$ over the range of $55 \mathrm{~km}$.

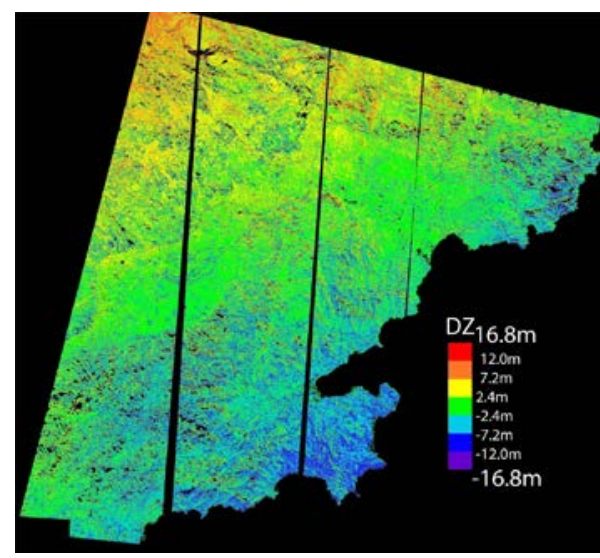

Figure 2. Color coded height differences ZY3 DSM, based on affine bias correction, against reference DSM from IGN

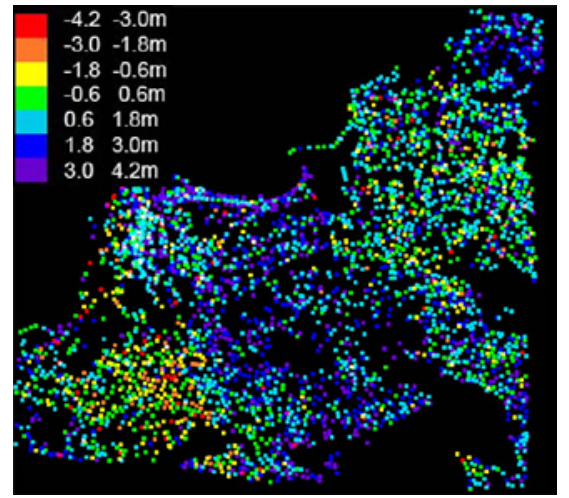

Figure 3. Color coded height differences Pléiades Zonguldak, orientation based on bias correction by affine transformation
The Pléiades height model (figure 3) does not show an obvious tilt, but a tendency of low frequency deformation. It has to be taken into account that this height model has an unusual short base with a base to height relation of 1:4.5, enlarging small image deformations to larger height errors.

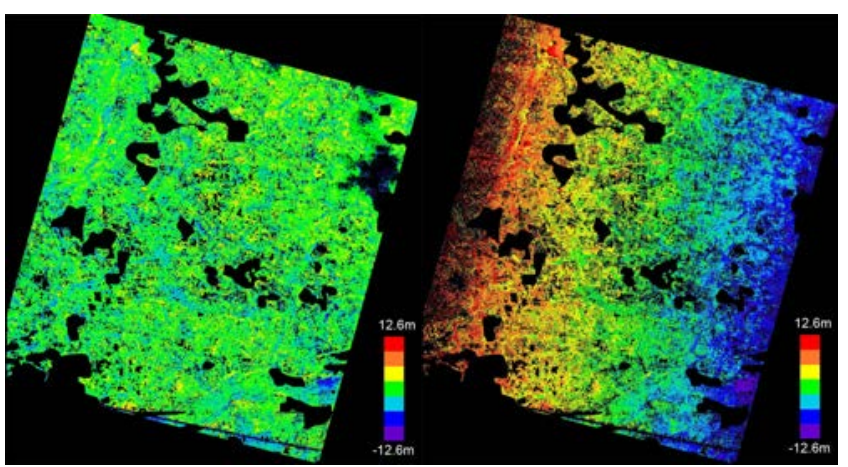

Figure 4. Color coded height differences Cartosat-1 Warsaw, left orientation by bias correction with affine transformation, right orientation by bias correction with shift; black areas masked out forest

In the Cartosat-1 height model (figure 4) the forest areas are masked out due to the reference digital terrain model (DTM) with the points on bare ground. Figure 4 shows on left hand side the color coded height differences of the height model based on RPC-orientation with affine bias correction and on right hand side the differences based on RPC-orientation just with shift correction. The affine parameters of the orientation are highly significant with Student test values (size of parameter divided by its own standard deviation) in the average exceeding the value of 20 .

\begin{tabular}{|l|c|c|c|c|c|c|}
\hline $\begin{array}{c}\text { ZY3 } \\
\text { affine }\end{array}$ & SZ & NMAD & $\begin{array}{c}\text { SZ } \\
<0.1\end{array}$ & $\begin{array}{c}\text { NMAD } \\
<0.1\end{array}$ & $\begin{array}{c}\text { Tilt } \\
\text { X }\end{array}$ & $\begin{array}{c}\text { Tilt } \\
\text { Y }\end{array}$ \\
\hline Original & 5.20 & 4.93 & 4.30 & 4.16 & 2.75 & -9.67 \\
\hline Leveled & 4.73 & 3.79 & 3.41 & 2.84 & - & - \\
\hline final & 4.72 & 3.71 & 3.41 & 2.83 & - & - \\
\hline
\end{tabular}

Table 8: Accuracy figures and DSM-tilt over full range [m], ZY3 DSM against reference DSM; orientation by bias correction with affine transformation; $<0.1=$ for slope below $0.1\left(10 \%\right.$ or $\left.5.7^{\circ}\right)$; final = leveled DSM corrected by low frequency systematic height errors

\begin{tabular}{|l|c|c|c|c|c|c|}
\hline $\begin{array}{c}\text { ZY3 } \\
\text { shift }\end{array}$ & SZ & NMAD & $\begin{array}{c}\text { SZ } \\
<0.1\end{array}$ & $\begin{array}{c}\text { NMAD } \\
<0.1\end{array}$ & $\begin{array}{c}\text { Tilt } \\
\text { X }\end{array}$ & $\begin{array}{c}\text { Tilt } \\
\text { Y }\end{array}$ \\
\hline Original & 5.47 & 5.19 & 4.43 & 4.32 & 2.72 & -9.46 \\
\hline Leveled & 4.73 & 3.79 & 3.42 & 2.84 & - & - \\
\hline final & 4.70 & 3.70 & 3.39 & 2.77 & - & - \\
\hline
\end{tabular}

Table 9: Accuracy figures and DSM-tilt over full range [m], ZY3 against reference DSM; orientation by bias correction by shift; $<0.1$ = for slope below 0.1

\begin{tabular}{|c|c|c|c|c|c|c|}
\hline $\begin{array}{c}\text { affine, } \\
\text { AW3D30 }\end{array}$ & SZ & NMAD & $\begin{array}{c}\text { SZ } \\
<0.1\end{array}$ & $\begin{array}{c}\text { NMAD } \\
<0.1\end{array}$ & $\begin{array}{c}\text { Tilt } \\
\mathrm{X}\end{array}$ & $\begin{array}{c}\text { Tilt } \\
\mathrm{Y}\end{array}$ \\
\hline Original & 5.27 & 2.84 & 4.40 & 4.18 & 6.37 & 7.59 \\
\hline Leveled & 4.86 & 4.11 & 3.10 & 2.53 & - & - \\
\hline final & 4.86 & 4.10 & 3.10 & 2.53 & - & - \\
\hline
\end{tabular}

Table 10: Accuracy figures and DSM-tilt over full range [m], ZY3 against AW3D30 DSM; orientation by bias correction with affine transformation; $<0.1$ = for slope below 0.1 


\begin{tabular}{|l|c|c|c|c|c|c|}
\hline $\begin{array}{c}\text { Pléiades } \\
\text { affine }\end{array}$ & SZ & NMAD & $\begin{array}{c}\text { SZ } \\
<0.1\end{array}$ & $\begin{array}{c}\text { NMAD } \\
<0.1\end{array}$ & $\begin{array}{c}\text { Tilt } \\
\mathrm{X}\end{array}$ & $\begin{array}{c}\text { Tilt } \\
\mathrm{Y}\end{array}$ \\
\hline Original & 1.68 & 1.57 & 1.55 & 1.55 & 0.53 & 1.35 \\
\hline Leveled & 1.68 & 1.60 & 1.62 & 1.54 & - & - \\
\hline final & 1.63 & 1.62 & 1.56 & 1.53 & - & - \\
\hline
\end{tabular}

Table 11: Accuracy figures and DSM-tilt over full range [m], Pléiades against reference DSM; $<0.1$ = for slope below 0.1 ; orientation by bias correction with affine transformation

\begin{tabular}{|l|c|c|c|c|c|c|}
\hline $\begin{array}{c}\text { Cartosat } \\
\text { affine }\end{array}$ & SZ & NMAD & $\begin{array}{c}\text { SZ } \\
<0.1\end{array}$ & $\begin{array}{c}\text { NMAD } \\
<0.1\end{array}$ & $\begin{array}{c}\text { Tilt } \\
\text { X }\end{array}$ & $\begin{array}{c}\text { Tilt } \\
\text { Y }\end{array}$ \\
\hline Original & 2.52 & 2.28 & 2.52 & 2.19 & -0.38 & -1.13 \\
\hline Leveled & 2.50 & 2.23 & 2.50 & 2.18 & - & - \\
\hline final & 2.50 & 2.23 & 2.50 & 2.18 & - & - \\
\hline
\end{tabular}

Table 12: Accuracy figures and DSM-tilt over full range [m], Cartosat-1 against reference DSM; $<0.1$ = for slope below 0.1 ; orientation by bias correction with affine transformation

\begin{tabular}{|l|c|c|c|c|c|c|}
\hline $\begin{array}{c}\text { Cartosat } \\
\text { shift }\end{array}$ & SZ & NMAD & $\begin{array}{c}\text { SZ } \\
<0.1\end{array}$ & $\begin{array}{c}\text { NMAD } \\
<0.1\end{array}$ & $\begin{array}{c}\text { Tilt } \\
\text { X }\end{array}$ & $\begin{array}{c}\text { Tilt } \\
\text { Y }\end{array}$ \\
\hline Original & 5.19 & 5.92 & 5.19 & 5.55 & 21.31 & -0.44 \\
\hline Leveled & 2.51 & 2.24 & 2.51 & 2.18 & - & - \\
\hline final & 2.49 & 2.23 & 2.47 & 2.18 & - & - \\
\hline
\end{tabular}

Table 13: Accuracy figures and DSM-tilt over full range [m], Cartosat-1 against reference DSM; $<0.1$ = for slope below 0.1 ; orientation by bias correction with shift

As accuracy figures for the height models the standard deviation of height differences (SZ) and the normalized median deviation (NMAD) (Höhle and Höhle 2009) are used.

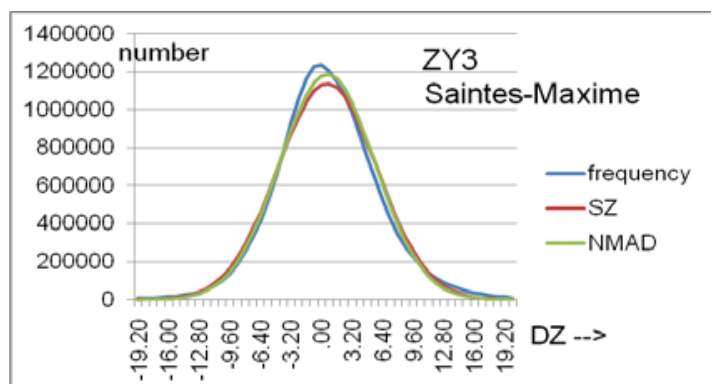

Figure 5. Frequency distribution of height differences ZY3 against reference with overlaid normal distribution for standard deviation and NMAD

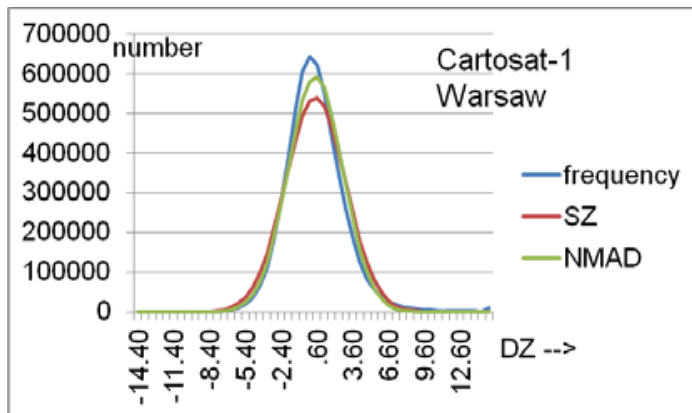

Figure 6. Frequency distribution of height differences, Cartosat-1 Warsaw

Typical relations between the frequency distribution of height differences and normal distribution based on standard deviation and NMAD are shown in figures 5 and 6. NMAD fits to the frequency distribution with an accuracy of $3.1 \%$ respectively $3.8 \%$, while this is for the standard deviation $3.8 \%$ respectively
5.5\%. Also for other data sets handled by the author NMAD fits better to the frequency distribution as the standard deviation. That means NMAD is a better accuracy figure for describing the frequency distribution of the height model differences.

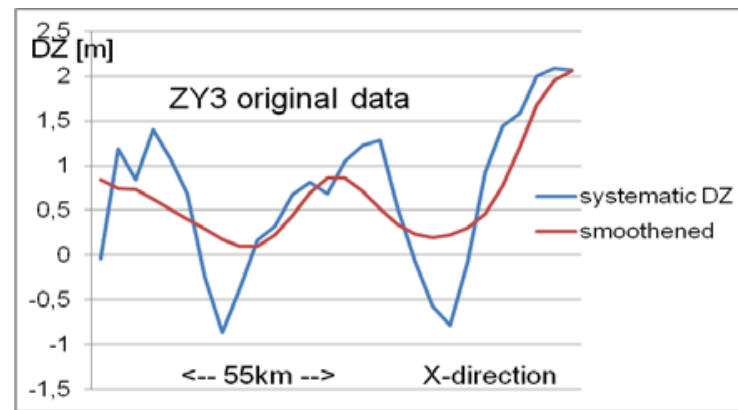

Figure 7. Systematic height errors in X-direction, SaintesMaxime, original ZY3 DSM

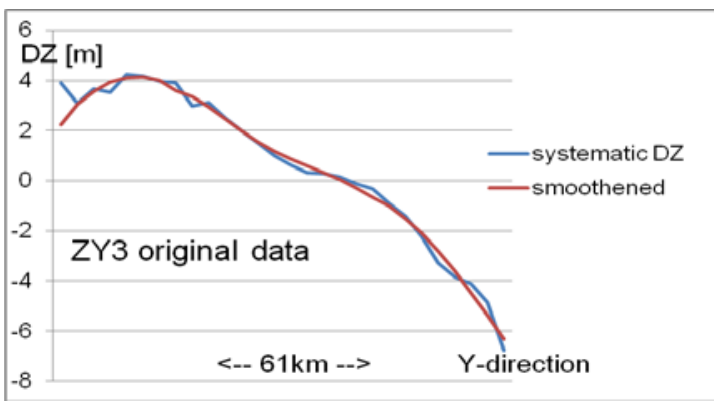

Figure 8. Systematic height errors in Y-direction, SaintesMaxime, original ZY3 DSM

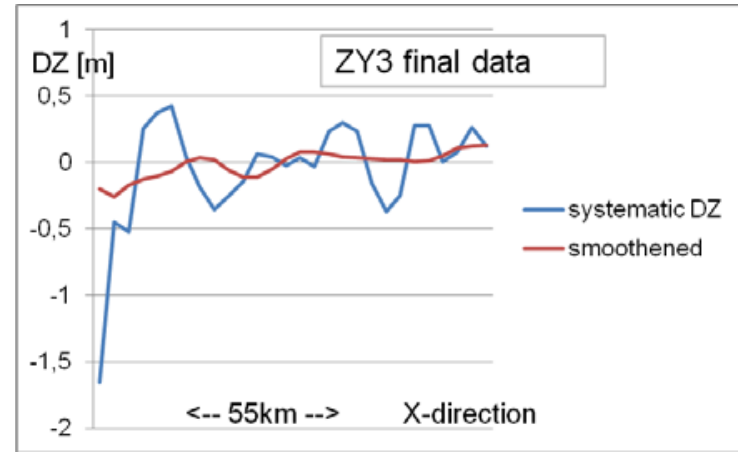

Figure 9. Systematic height errors in X-direction, SaintesMaxime after leveling ZY3 DSM and reduction of low frequency systematic errors

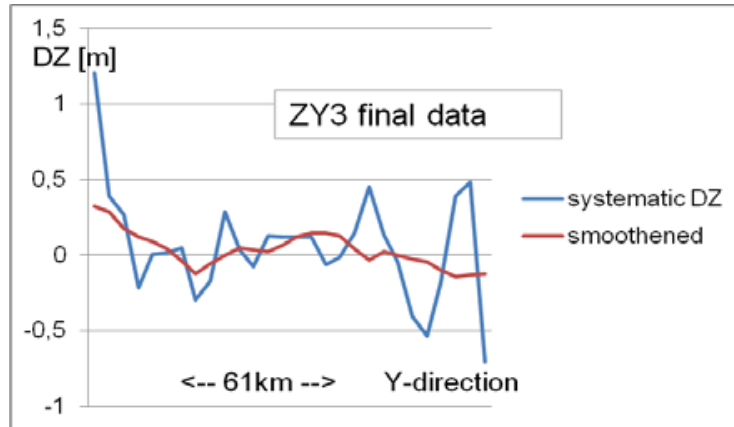

Figure 10. Systematic height errors in Y-direction, SaintesMaxime after leveling ZY3 DSM and reduction of low frequency systematic errors 


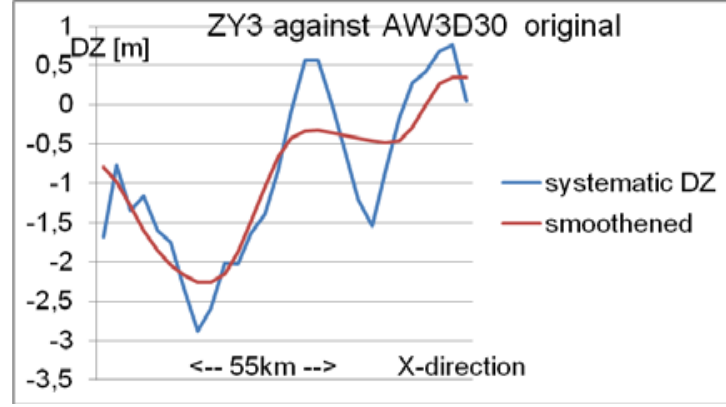

Figure 11. Systematic height errors in X-direction, SaintesMaxime, ZY3 DSM against AW3D30 DSM

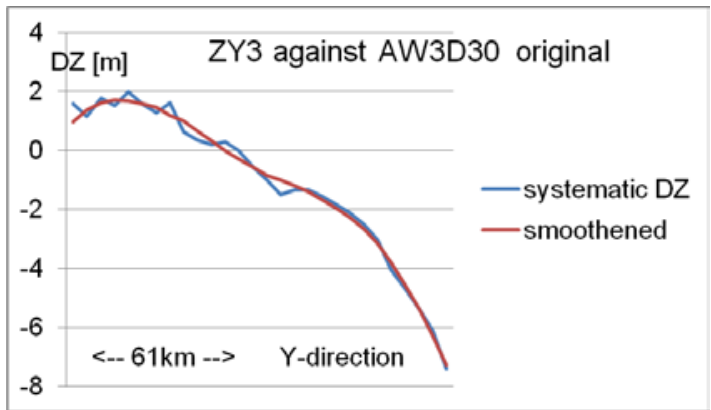

Figure 12. Systematic height errors in Y-direction, SaintesMaxime, ZY3 DSM against AW3D30 DSM

For numerical analysis and for correction of the DSM tilt and low frequency systematic height errors, the height differences have been averaged in 30 groups in $\mathrm{X}$ - and also Y-direction, shown in figures 7 to 12 as "systematic DZ". For the reduction of local effects, which may be caused by height change of the vegetation or not respected forest in case of a reference DTM, the systematic errors have been smoothened by moving polynomial of $2^{\text {nd }}$ degree using 5 up to 15 neighbored groups here 15 groups have been used. The smoothened values are shown as red lined labeled as "smoothened". The smoothing uses the number of values in each group as weight, explaining some larger discrepancies especially at both ends of the systematic errors where the number of values usually is lower as in the center.

The three here analyzed data sets from different satellite sensors have different characteristics. The ZY3 height models of Saintes-Maxime have a strong tilt independent upon RPCorientation with bias correction by affine transformation or by shift. The accuracy of the original height model is a little better if the orientation is determined by RPC-orientation with affine bias correction as with shift bias correction. After leveling of the height model the type of used orientation has no influence (tables 8 and 9). The ZY3 height model has a strong tilt in Ydirection (in flight direction); while in $\mathrm{X}$-direction (across flight direction) a low frequency undulation can be seen. After leveling of the height models and elimination of the low frequency errors the remaining smoothened systematic height errors are negligible in relation to the achieved accuracy. The accuracy numbers are reduced by the leveling of the height models while the elimination of the low frequency systematic errors only has a limited influence to the accuracy numbers (tables 8 - 10). Nevertheless low frequency height errors as obvious in figure 7 , with a size exceeding $1 \mathrm{~m}$, have to be eliminated for an NMAD for a terrain slope up to $0.1\left(\sim 6^{\circ}\right)$ of $2.8 \mathrm{~m}$. This accuracy is a very good result for $3.4 \mathrm{~m}$ GSD images and a base to height relation of 1:1.15 - it corresponds to an $\mathrm{x}$ parallax of 0.7 pixels.
The ZY3 DSM has been compared also with the AW3D30 DSM (table 10). AW3D30 (Takaku et al. 2014), based on ALOS PRISM, has against SRTM the advantage of 1 arcsec point spacing ( $\sim 30 \mathrm{~m}$ at equator) which is not available by SRTM in the area of Turkey. In addition the ALOS PRISM data are more actual as SRTM and the up to now by the author analyzed AW3D30 DSMs are more accurate as SRTM DSMs. The standard deviation and NMAD of the height differences of ZY3-DSM against French reference DSM and ZY3-DSM against AW3D30 DSM are nearly the same (tables 8 and 10). Also the shape of the systematic height differences (figures 7 against 11 and 8 against 12) is very similar, only the model tilt is different. After tilt compensation for areas with a slope $<0.1$ $\mathrm{SZ}$ and NMAD are even smaller as in relation to the reference DSM from IGN, but this has to respect the definition of terrain slope, which is different for $10 \mathrm{~m}$ against $27 \mathrm{~m}$ point spacing. Of course the AW3D30 DSM is not as accurate as the reference DSM from IGN, which has a standard deviation in the range of $20 \mathrm{~cm}$. Nevertheless the object definition by ALOS PRISM with $2.5 \mathrm{~m}$ GSD and a base to height relation between forward and backward camera of 1:1.0 is very similar to the object definition by ZY3, causing such result. Finally this demonstrates that for the improvement of the height models by tilt and low frequency deformation not a very precise reference DSM is required, the AW3D30 DSM is satisfying.

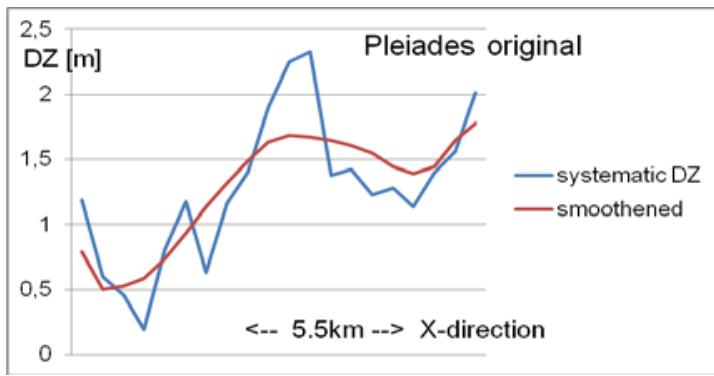

Fig. 11. Systematic height errors in X-direction, Pléiades Zonguldak original DSM against reference DTM

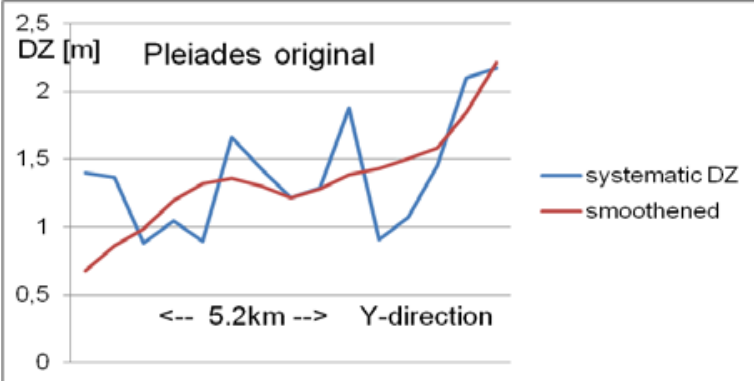

Figure 12. Systematic height errors in Y-direction, Zonguldak, original Pléiades DSM against reference DTM

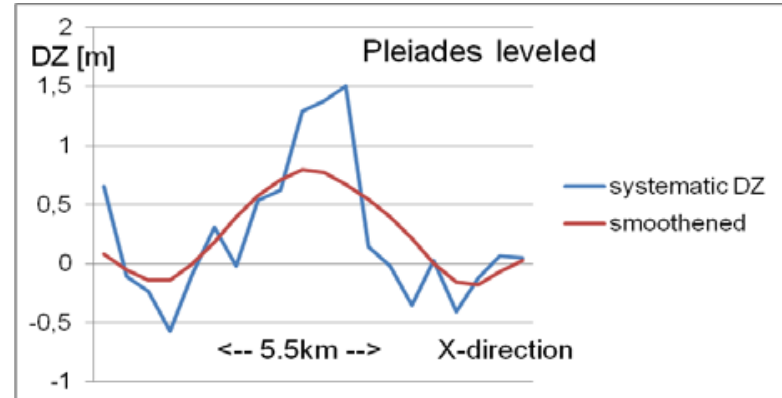

Figure 13. Systematic height errors in X-direction, Zonguldak, leveled Pléiades DSM against reference DTM 


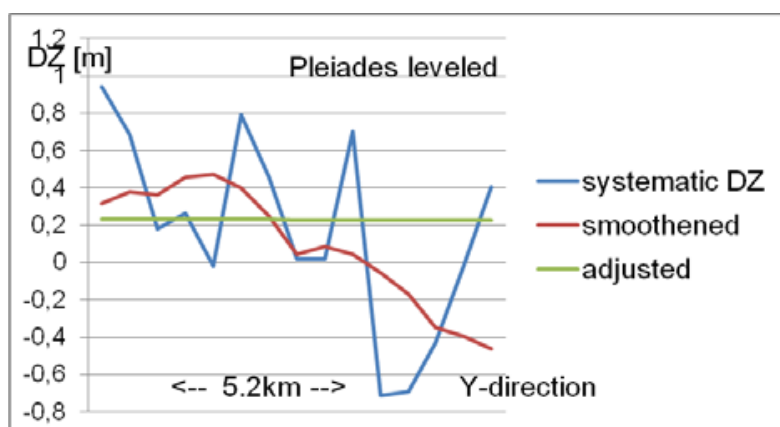

Figure 14. Systematic height errors in Y-direction, Zonguldak, leveled Pléiades DSM against reference DTM

The bias correction affine parameters of the Pléiades RPCorientation with 170 GCP, with the exception of the shift parameters, just have average Student test values of 2.7. Its influence to the orientation is limited. The accuracy numbers of the Pléiades DSM based on RPC-orientation with affine transformation and just with shift are nearly identical, as also the DSM tilt values, so only table 11 shows the accuracy numbers and tilt of the Pléiades DSM. The model tilt is not as large as for ZY3 and Cartosat, nevertheless in X-direction a low frequency systematic error can be seen, which is also obvious in figure 3 . The accuracy numbers for the original DSM are not improved by height model leveling, but they are slightly improved by the elimination of the low frequency systematic height errors (table 11). The NMAD of $1.53 \mathrm{~m}$ for terrain slope not exceeding 0.1 corresponds to 0.5 pixels of the original $0.7 \mathrm{~m}$ GSD or 0.7pixels for the delivered $0.5 \mathrm{~m}$ GSD. The image quality as also the reached accuracy justifies a distribution of Pléiades images with 0.5m GSD (Jacobsen et al. 2014).

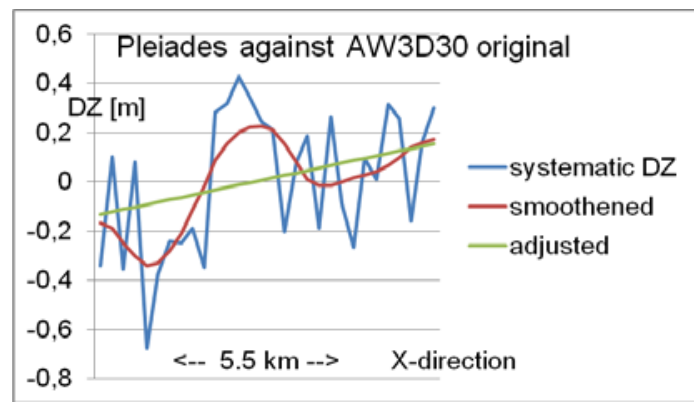

Fig. 15. Systematic height errors in X-direction, Zonguldak, original Pléiades DSM against AW3D30

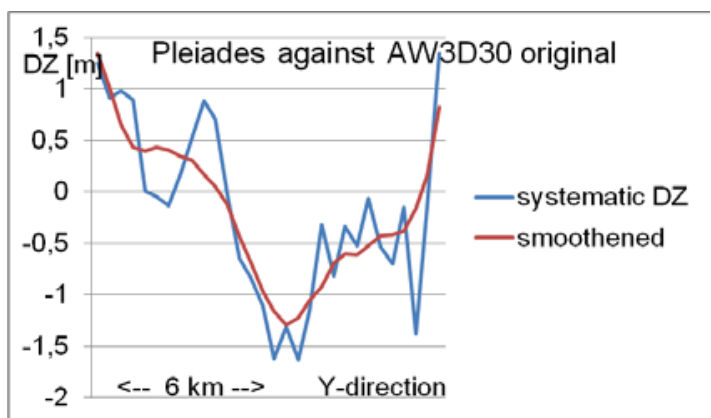

Fig. 16. Systematic height errors in Y-direction, Zonguldak, original Pléiades DSM against AW3D30

The reference DTM used for Pléiades DSM has only a limited number of not equal distributed points (figure 3 ), due to this reason the Pléiades DSM has been compared also with the AW3D30 DSM (figure 17).

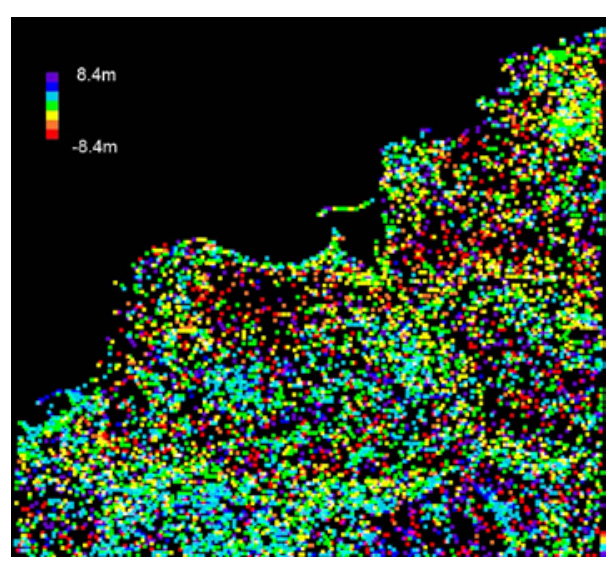

Fig. 17: Color coded height differences Pléiades DSM Zonguldak against AW3D30 DSM

\begin{tabular}{|l|c|c|c|c|c|c|}
\hline & SZ & NMAD & $\begin{array}{c}\text { SZ } \\
<0.1\end{array}$ & $\begin{array}{c}\text { NMAD } \\
<0.1\end{array}$ & $\begin{array}{c}\text { Tilt } \\
\text { X }\end{array}$ & $\begin{array}{c}\text { Tilt } \\
\text { Y }\end{array}$ \\
\hline Original & 2.75 & 2.37 & 2.11 & 1.84 & 0.30 & 0.86 \\
\hline Leveled & 2.64 & 2.30 & 2.04 & 1.92 & - & - \\
\hline final & 2.62 & 2.25 & 1.98 & 1.83 & - & - \\
\hline
\end{tabular}

Table 14: Accuracy figures and DSM-tilt over full range [m] Pléiades DSM against AW3D30 DSM, Zonguldak

The accuracy numbers of the height differences between Pléiades DSM and AW3D30 are astonishing small (table 14). The tilt of Pléiades DSM against the reference DTM, is slightly smaller. The low frequency systematic differences for the $\mathrm{X}$ direction agrees very well (compare figure 15 with figure 11). In Y-direction the smoothened differences are different - this is also obvious by the comparison of the color coded height differences (figures 3 and 17). It has to be respected that for the comparison of the Pléiades DSM against the reference DTM only 1470 points are available and for the comparison with AW3D30 DSM 3103 points. For an analysis of height models this is a small number which cannot guarantee that the determined deformations may not be caused by changes in object space.

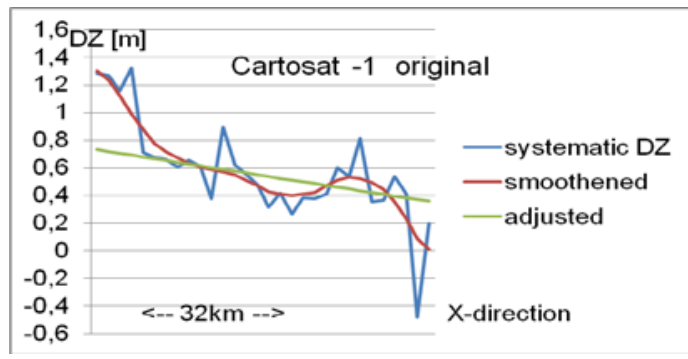

Fig. 17. Systematic height errors in X-direction, Warsaw, filtered Cartosat-1 DSM against reference DTM

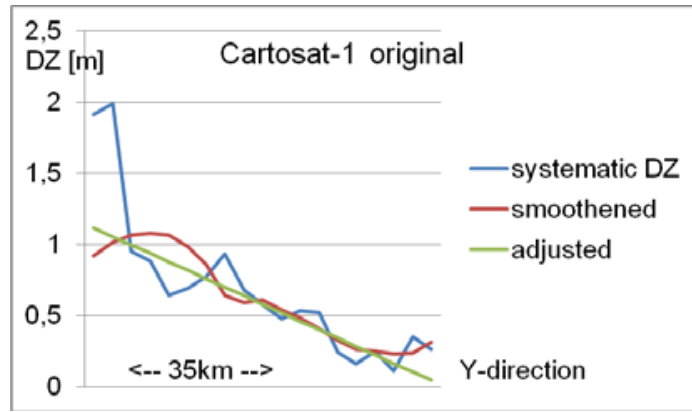

Fig. 18. Systematic height errors in Y-direction, Warsaw, filtered Cartosat-1 DSM against reference DTM 


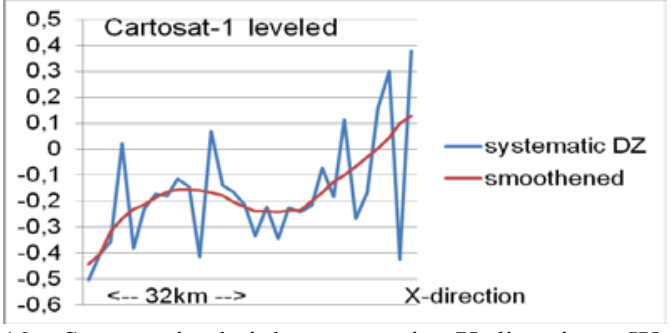

Fig. 19. Systematic height errors in X-direction, Warsaw, leveled Cartosat-1 DSM against reference DTM

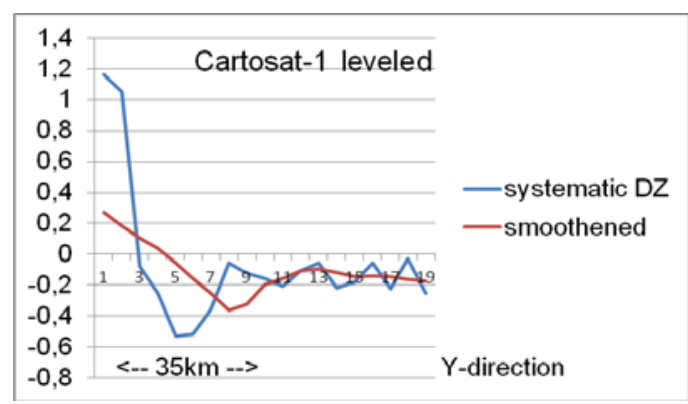

Fig. 20. Systematic height errors in Y-direction, Warsaw, Leveled Cartosat-1 DSM against reference DTM

As obvious in tables 5, 12 and 13 and figure 1, the Cartosat-1 data set Warsaw requires an RPC-orientation with bias correction by affine transformation; the orientation with bias correction by shift leads to a very strong DSM tilt. Nevertheless after leveling the results with both bias corrections, nearly the same results are reached. The accuracy figures are only slightly improved by leveling of the DSM based on orientation with affine bias correction, the elimination of the limited low frequency systematic errors does not lead to further improvement (see also figures 17 up to 20).

The NMAD of $2.18 \mathrm{~m}$ corresponds for the base to height relation of 1:1.6 and 2.5m GSD to 0.55 pixels.

\section{CONCLUSION}

The analyzed height models based on ZY3, Pléiades 1A and Cartosat- 1 stereo configurations are influenced by tilt and low frequency systematic errors. The model tilt for ZY3 and Cartosat-1 DSMs depends upon the RPC bias orientation by affine transformation or by shift. Better results have been achieved with bias orientation by affine transformation. Low frequency height errors have a not negligible effect to the ZY3 DSM while for all height models a leveling is required.

It is satisfying to use standard free of charge available DSMs for the analysis and correction of systematic DSM errors. With AW3D30 DSMs very good results have been reached, better results as by SRTM DSMs. It has to be guaranteed, that no influence of the height model point definition as DSM or DTM is misinterpreted as low frequency height error - forest areas have to be masked out and the generated DSM should be filtered to avoid an effect by small tree groups and buildings. The analyzed DSM should have enough height points to avoid a misinterpretation of random points as low frequency systematic height errors.

\section{REFERENCES}

d'Angelo, P., 2013. Automatic orientation of large multitemporal satellite image blocks. In: Proceedings of International Symposium on Satellite Mapping Technology and Application 2013

Grodecki, J., 2001: IKONOS stereo feature extraction - RPCapproach, ASPRS Annual Conference, St. Louis, USA, 2001

Höhle, J \& M Höhle, 2009. Accuracy assessment of digital elevation models by means of robust statistical methods, ISPRS Journal of Photogrammetry and Remote Sensing, 64, pp. 398406

Jacobsen, K., 2003: Geometric Potential of IKONOS- and QuickBird-Images, in D. Fritsch (Ed.) Photogrammetric Weeks '03, pp 101-110, Wichmann Verlag ISBN 3-87907-397-X, and GIS Geo-Informations-Systeme 9/2003, pp33 -39

Jacobsen, K., Topan, H., Cam, A., Özendi, M., Oruc, M., 2014: Radiometric and Geometric Characteristics of Pléiades Images, ISPRS Com I symposium, Denver, ISPRS Archives - Volume XL-1

Jacobsen, K., 2015: Geometric potential of Pléiades models with small base length, EARSeL Symposia Stockholm 2015

Jacobsen, K., Topan, H., 2015: DEM generation with short base length Pléiades Triplet, PIA/HRSI workshop Munich 2015, IntArchPhRS Vol XL-3/W2

Takaku, J., Tadono, T., Tsutsui, K., 2014 : Generation of High Resolution Global DSM from ALOS PRISM, The International Archives of the Photogrammetry, Remote Sensing and Spatial Information Sciences, pp.243-248, Vol. XL-4, ISPRS TC IV Symposium, Suzhou, China, 2014.

Yong-hua Jiang et al. 2015: Geometric Calibration and Accuracy Assessment of ZiYuan-3 Multispectral Images, IEEE Xplore 2015

Zhang, Guo et al. 2015: Geometric accuracy improvement and assessment of Chinese high-resolution optical satellites, IEEE JSTARS, 2015.2429151 